\title{
Febre em crianças: procura de pais por serviços médicos de emergência
}

\author{
Fever in children: parents' search for urgent and emergency services
}

Pedro Jose Pitoli (https://orcid.org/0000-0001-8395-4720) ${ }^{1}$

Brenda Katheryne Duarte (https://orcid.org/0000-0003-2581-9015) ${ }^{1}$

Andressa Amorim Fragoso (https://orcid.org/0000-0001-8442-5863) ${ }^{1}$

Daniela Garcia Damaceno (https://orcid.org/0000-0001-8656-009X) ${ }^{2}$

Maria José Sanches Marin (https://orcid.org/0000-0001-6210-6941) ${ }^{1}$

${ }^{1}$ Faculdade de Medicina de

Marília. R. Monte Carmelo

800, Fragata. 17519-

030 Marília SP Brasil.

pedropitoli@gmail.com

${ }^{2}$ Universidade Estadual

Paulista Júlio de Mesquita

Filho. Botucatu SP Brasil.

\begin{abstract}
The study analyses the understanding of parents or guardians of children aged zero to five years old about fever, the conduct and care for this condition, carried out in the urgent and emergency service. The qualitative research, which used the thematic analysis technique, was carried out through interviews with 14 parents or guardians who sought an urgent and emergency Pediatric Service in a municipality in the countryside of São Paulo for this reason. The experiences of parents who seek these services are associated to three main topics: fear of fever; care for a febrile child; and the experience of care at the urgent and emergency services. It was identified that an exaggerated fear of fever predominates and that parents / guardians feel safe regarding the existing technology found in urgent and emergency services. The adopted precautions, however, are not always those recommended for the existing situation.
\end{abstract}

Key words Child health, Fever, Child care, Parents, Emergency medical service
Resumo O estudo analisa a compreensão de pais ou responsáveis por crianças de zero a cinco anos acerca da febre, da condução e do atendimento desse quadro, realizado no serviço de urgência e emergência. A pesquisa qualitativa, por meio da técnica de análise temática, foi feita a partir de entrevistas com 14 pais ou responsáveis que procuraram um serviço pediátrico de urgência e emergência em um município do interior de São Paulo por esse motivo. Os dados coletados foram analisados por meio da técnica de análise temática. As experiencias dos pais que procuram esses serviços relacionam-se a três temas principais: temor da febre; os cuidados com criança febril; e a experiência de atendimento no serviço de urgência e emergência. Identificou-se que o medo exagerado da febre prevalece e que os pais/responsáveis se sentem seguros frente à tecnologia existente nos serviços de urgência e emergência. Os cuidados adotados, todavia, nem sempre são os recomendados para a situação apresentada.

Palavras-chave Saúde da criança, Febre, Cuidado da criança, Pais, Serviços médicos de emergência 


\section{Introdução}

A febre é um achado comum na infância. Aproximadamente, de 20 a $40 \%$ das crianças até os cinco anos são levadas ao atendimento médico por causa de estado febril. Os conceitos sobre a febre e as formas de manejo, embora ao longo do tempo tenham passado por distintas compreensões, desde a época de Hipócrates, 400 anos aC, têm sido considerados benéficos e terapêutica, visto ser um evento necessário à restauração do estado de saúde ${ }^{1,2}$.

Em 1876, o médico e fisiologista francês Claude Bernard realizou estudo, em que constatou que animais morriam quando a temperatura de seus corpos aumentava de cinco a seis graus Celsius acima do normal. Deste modo, o conceito da febre como salutar ao organismo deixou de ser considerado em sua totalidade ${ }^{3}$.

Com a ruptura do paradigma, esforços foram empreendidos para compreendê-la e, portanto, controlá-la. Assim, ao final do século XIX, novas drogas antipiréticas foram descobertas e passaram a ser utilizadas de modo corriqueiro em todo o mundo. Frente a esse contexto, houve aumento de procura dos serviços de saúde e consequentes dificuldades da equipe de saúde em realizar a abordagem diagnóstica e terapêutica de quadros febris. Somente em meados da década de 1980, alguns critérios, como os de Boston, Milwaukee, Filadélfia e Rochester, foram elaborados e serviram de base para diretrizes e protocolos das sociedades médicas, utilizados na prática clínica com atualizações até os dias de hoje .

Por essas diretrizes, o principal ponto para avaliação inicial de uma criança, independente do grau de febre, é o comprometimento de seu estado geral, avaliado por parâmetros, como frequência cardíaca, respiratória, tempo de enchimento capilar, grau de hidratação, atividade e responsividade aos estímulos, uma vez que, isoladamente, a febre não é fator de risco para se constatar a gravidade da doença $a^{5}$.

Encontram-se poucas evidências da relação da febre alta com a gravidade de doenças. Isso foi corroborado em amplo estudo realizado na Austrália, com 16.000 casos de crianças com febre, demonstrando baixo valor preditivo nessa relação, pois se constatou que menos de uma, a cada 250 crianças febris menores de cinco anos, evolui para doença invasiva ${ }^{6}$, sendo esse risco próximo de $3,2 \%$.

Enfatiza-se, ainda, que, a partir da introdução das vacinas contra Haemophilus tipo b (HIB) e vacina contra Streptococcus pneumoniae, houve diminuição importante na ocorrência das doenças de maior potencial de gravidade, uma vez que esses agentes têm sido os principais responsáveis por doenças, como Pneumonia, Meningite e Sepse, na faixa etária até três anos de idade ${ }^{8}$.

Mesmo frente a tais evidências, essa queixa ainda permanece como um dos principais motivos da procura de serviços de urgência e emergência em Pediatria, pois os pais percebem a febre como doença e fator de risco para complicações maiores - convulsões ou dano cerebral. A percepção equivocada dos fatos ocorre, muitas vezes, por a febre causar aparência física desagradável na criança, gerando sentimentos de medo, ansiedade e insegurança frente a qualquer novo episódio febril. Assim, esses conceitos incorretos geram temor descrito como febrefobia, termo utilizado para definir os medos irrealistas em relação ao caso $^{9-11}$.

Ao pautar-se em práticas e crenças, a febre pode ser entendida sob três enfoques: científico, científico pragmático e fóbico-temeroso. O primeiro é caracterizado pela resposta adaptativa e benéfica ao organismo; o segundo se define pelo reconhecimento da função da febre no organismo e, mesmo assim, sendo tratada pelos profissionais de saúde, devido ao conhecimento prévio sobre febre; o terceiro, o modelo fóbico-temeroso, é marcado por altos índices de ansiedade e medo frequente entre pais ou responsáveis ${ }^{12}$.

Essa disparidade conceitual sobre febre evidencia a necessidade do contínuo entendimento sobre o tema em diferentes realidades, visto que pode induzir a condutas inadequadas, mesmo sob orientação profissional. Assim, evidenciaram-se os seguintes questionamentos: Como pais ou responsáveis por crianças de zero a cinco anos compreendem a febre e o atendimento realizado no serviço de urgência e emergência? Quais as condutas que adotam na presença de um quadro febril?

Frente a esse contexto, a presente investigação tem como objetivo analisar a compreensão de pais ou responsáveis por crianças de zero a cinco anos acerca da febre, da sua condução e do atendimento ao quadro febril, realizado no serviço de urgência e emergência.

\section{Aspectos éticos}

O estudo respeitou os preceitos éticos relativos a pesquisas com seres humanos, estabelecidos pela Resolução no 510, de 7 de abril de 2016, do Conselho Nacional de Saúde. A proposta de investigação foi submetida ao Comitê de Ética e 
Pesquisa com Seres Humanos, da Faculdade de Medicina de Marília (FAMEMA) e aprovada. Os participantes da investigação assinaram o Termo de Consentimento Livre e Esclarecido. Na apresentação dos resultados, as entrevistadas foram designadas pela letra E, seguida de sequência numérica, conforme a ordem das entrevistas, de 1 a 14, para assegurar o anonimato.

\section{Tipo de estudo}

Trata-se de um estudo qualitativo, realizado a partir de entrevistas com pais ou responsáveis que levaram crianças ao serviço de urgência e emergência de um hospital pediátrico de um município interior do estado de São Paulo, Brasil.

\section{Cenário do estudo}

O hospital onde o estudo foi realizado é considerado referência para alta complexidade da Rede Regional de Atenção à Saúde, a qual conta com cinco microrregiões de saúde, com $62 \mathrm{mu}$ nicípios e atende a uma população estimada de 1.128.941 habitantes. O serviço de urgência e emergência realizou, no ano de 2016, 407 atendimentos a crianças com o diagnóstico de febre (CID de febre na ficha de atendimento).

\section{Fonte e coleta de dados}

Foram incluídos no estudo pais ou responsáveis de crianças com menos de cinco anos de idade que deram entrada no serviço de urgência e emergência com queixa de febre. Excluíram-se pais de crianças portadoras de alguma doença crônica, como as hematológicas e neoplasias, ou outro problema de saúde associado à febre.

A coleta de dados foi realizada pelos próprios pesquisadores, nos meses de setembro a novembro de 2018, em diferentes dias da semana e períodos do dia, com vista a obter diversificação amostral. Depois de constatado que a criança chegou ao serviço tendo como queixa principal a febre, o pai ou responsável foi convidado a participar da entrevista. Aqueles que aceitaram foram encaminhados para sala reservada, com a finalidade de manter privacidade e conforto.

As entrevistas foram realizadas com 14 pessoas, conforme roteiro contendo dados de identificação e questões abertas sobre o significado da febre, os motivos que as levaram a procurar o serviço de urgência e emergência, as condutas adotadas em relação à febre e a opinião a respeito do atendimento nesse serviço. As entrevistas, com duração média de 20 minutos, foram audiogravadas.

A amostra foi encerrada quando houve constatação da saturação teórica dos dados, momento da pesquisa no qual a coleta de novos dados não traria mais esclarecimentos para o tema estudado. Cita-se, ainda, que o ponto de saturação, assim como o tamanho da amostra, é resultante da heterogeneidade da população pesquisada. Neste tipo de pesquisa, preza-se a quantidade e a qualidade de dados, conceitos que se relacionam com seu volume e riqueza ${ }^{13}$.

\section{Organização e análise dos dados}

Os dados obtidos foram alvo da técnica de análise temática, método analítico qualitativo que busca identificar, analisar, relatar padrões (tema) e interpretar os achados. Nesse procedimento, o tema representa algo importante a ser abstraído dos dados em relação à pergunta de pesquisa e tem significado padronizado, podendo ou não aparecer com grande prevalência no conjunto das informações ${ }^{14}$. Mais especificamente, um tema representa um grupo de ideias e contém códigos comuns, com alto grau de generalidade que unifica ideias sobre o assunto investigado ${ }^{15}$.

A operacionalização da análise temática ocorre em seis fases que permitem sucessivas aproximações com os dados. Entretanto, não se trata de processo linear, em que uma fase precede a outra, sendo necessário aplicar a flexibilidade e ser exaustivo na interação com os dados para se obter percepções ricas e complexas ${ }^{14}$. Na proposição das fases a serem seguidas, inicialmente, coloca-se a familiaridade com os dados, conseguida pela imersão neles, por meio de leituras repetidas a fim de nos aproximarmos da profundidade e amplitude do conteúdo.

A segunda fase envolve a produção de códigos iniciais a partir dos dados. Esses códigos representam o conteúdo semântico ou latente que se refere ao segmento ou elemento mais básico do dado. A terceira fase, a procura por temas, é desenvolvida a partir da lista de códigos e abrange a triagem dos diferentes para compor temas potenciais. Na finalização, haverá coleção de temas e subtemas. $\mathrm{Na}$ fase quatro, momento de revisitar os temas, engloba-se o refinamento e ponderam-se os critérios de homogeneidade interna e heterogeneidade externa para a criação de mapa temático. Na sequência, os temas são definidos e nomeados, ou seja, é identificada a essência do assunto de cada tema. Na última fase, inicia-se a análise e a escrita do relatório ${ }^{14}$. 


\section{Resultados}

Em relação ao parentesco do entrevistado com a criança, foram entrevistadas 12 mães, um pai e uma avó. A idade variou de 25 a 39 anos, com predomínio de menores de 30 anos. O nível de escolaridade mais presente foi o ensino médio completo e, em seguida, o ensino médio incompleto. Em relação ao estado civil, houve presença de casadas, solteiras e amasiadas. Os participantes eram procedentes do próprio município e de cidades vizinhas ${ }^{16}$.

A partir da codificação inicial, foram realizados novos contatos com os dados, com vista à elaboração dos códigos e respectivos temas, resultando no mapa temático final. Conforme Figura 1, evidenciaram-se três temas: o temor da febre; os cuidados com a criança febril; e a experiência de atendimento no serviço de urgênica e emergência.

\section{O temor da febre}

$\mathrm{Na}$ análise do significado da febre, os entrevistados manifestaram temor à ela, por associá-la à infecção, à convulsão, às doenças graves, à crença na baixa imunidade da criança e demonstraram preocupação com sinais e sintomas que deixam a criança debilitada, chorosa, sem se alimentar, cansada e com dor. A febre é compreendida como sinal de que alguma coisa anormal está acontecendo no organismo da criança, mesmo com experiências prévias de que sua ocorrência tenha significado agravamento no estado de saúde, como se pode verificar nos fragmentos:

Que alguma coisa está acontecendo. Que não está normal. Alguma infecção pode está acontecendo com a criança. (E3)

Fico preocupada de subir muito e dar convulsão.(E1)

Ah, porque a febre faz convulsionar. Tenho medo. (E12)

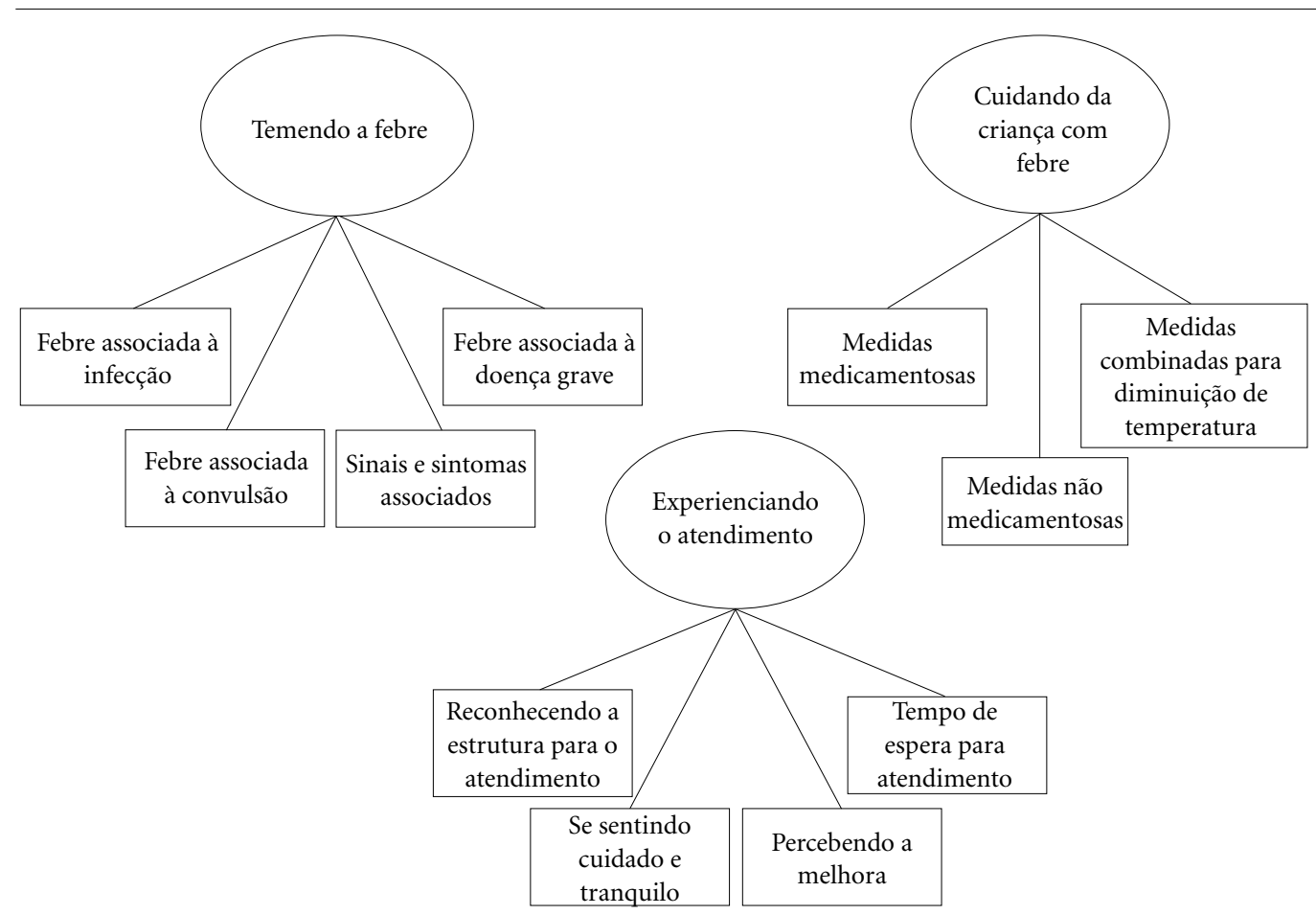

Figura 1. Mapa temático final, elaborado a partir das entrevistas com pais ou responsáveis pelas crianças atendidas no serviço de urgência e emergência. Marília, São Paulo, Brasil. 2019. 
Fico mais preocupada porque três meses de vida é muito pequeninha, não tem muita imunidade, não tem muita resistência, igual...maior... então, fico muito preocupada. E nunca aconteceu isso com ela. Primeira vez que ela teve febre. (E4)

Ah, essa gripe suína, misericórdia! Eu já penso, já fico preocupada por causa da gripe suína. A infecção dá em algum lugar. O povo fala, acho que um médico falou: "ah, está com febre, está com infecção". Procurar saber... que nem da outra vez que ele ficou aqui. Não achou nada, sei lá. (E1)

Você vê que a criança fica com dificuldade, não quer mamar, fica só chorando e fica.... deixa eu ver como que ela fica, fica muito cansadinha sabe? ...ela não é de chorar. (E4)

E aí, eu, como mãe, percebia que ela estava mais amoada, queria só colo. (E6)

\section{Os cuidados da criança febril}

No manejo da febre em crianças, os entrevistadados utilizavam medidas medicamentosas, não medicamentosas, bem como a associação de ambas.

Quanto às medidas medicamentosas, os entrevistados relataram a utilização do ibuprofeno isoladamente e, em caso de persistência do quadro febril, intercalavam com a dipirorna. Além disso, foi introduzido, também, o paracetamol em dose dobrada. A associação da dipirona com o paracetamol também foi relatada. Houve também a preocupação em não medicar a criança, ao compreender que, ao cessar a febre, o serviço de urgência e emergência poderia não atendê-la.

Com o ibuprofeno, passava. Era para ela tomar de oito em oito horas. Então nesse intervalo, dessas oito em oito, se ela tivesse febre, então eu dava a dipirona. Teve uma vez que estava muito forte, acho que foi na sexta, que eu dei de quatro em quatro horas. Dei a dipirona, não cortou, daí passou quatro horas, eu dei paracetamol. Só que eu até dei em dose dobrada porque sete gotinhas não iam fazer efeito para febre. (E2)

Eu dei a quantidade de gotas que era o peso dela. São onze quilos. Eu dei onze gotas de paracetamol. Aí ajudou a abaixar a febre. (E9)

Eu dei dipirona e também paracetamol. A avó deu também para intercalar. (E10)

Na hora, eu achei que se eu desse remédio para ela, não ia ser atendida aqui. /P: Tá.../E: Ia chegar, já ia está abaixada a febre, não ia me dar um atendimento que eu precisava ter, por motivo dela não estar com febre. (E14)

As medidas não medicamentosas usadas pelos responsáveis incluíram banho morno, uso de pano molhado na testa da criança e retirada de agasalhos. $\mathrm{O}$ banho com gotas de alcool também foi mencionado.

Antes, eu coloquei um pano molhado na testa dele. Porque no momento, eu estava sem medicação. (E3)

Aí, eu dou um banhinho de água morna nela. Até melhorar. Caso tudo isso não melhore, é onde eu trago no pronto socorro. (E8)

Dou banho com água morna. E, se tiver muito alta a febre, duas gotinhas de álcool na água. O banho é na banheira com água morna na banheira e deixo esfriar junto com a água. (E11)

Os responsáveis também indicaram que utilizavam medidas combinadas para o controle da febre.

Eu dou banho, medico, abaixa, mas depois volta. (E4)

Eu dou dipirona. E, além da dipirona, eu o coloquei no banho. (E9)

\section{A experiência do atendimento}

No que se refere ao atendimento do serviço de urgência e emergência, os entrevistados indicaram estrutura favorável, que perceberam melhora, sentiram-se cuidados e tranquilos. Entretanto alguns apontaram a demora no atendimento.

Quanto à estrutura favorável ao atendimento, verbalizaram que possibilita uma avaliação detalhada da situação, bem como bom atendimento em caso de agravamento do quadro, o que lhes proporcionava segurança, conforme explícito no seguinte fragmento de fala.

Mas, aqui, é uma coisa mais assim... que eles podem ter a profundidade do caso se ele tiver com alguma coisa. Tem UTI, se caso ocorrer alguma coisa mais grave. Então, aqui tem estrutura maior do que lá no PA. Dá uma segurança. (E10)

$\mathrm{O}$ atendimento da criança com febre no serviço de urgência e emergência, segundo se percebe, traz a sensação de a criança estar sendo cuidada, de estar tomando a medicação certa e fazendo exames. Isso tudo contribui para melhora do quadro, ou seja, para o desaparecimento da febre, trazendo-lhes tranquilidade. Além disso, os responsáveis mantinham a expectativa de que essa melhora iria continuar, conforme falas que seguem.

Eles falam direitinho o que ela tem realmente. Cuida. Dá o remédio, passa o remédio certo. (E4)

Então, assim, ela está bem. Exame de sangue dela, de urina... tem que melhorar um pouquinho mais. Mas, já fico mais tranquila. (E6) 
Melhor, já melhorou bastante. Já deu uma melhorada. A febre sumiu, só tem umas dorzinhas, mas a expectativa é de melhora completamente. Essa é nossa esperança que melhora. (E7)

Para os participantes do estudo, um inconveniente do atendimento no serviço de urgência e emergência refere-se à demora para isso acontecer.

Demorou um pouquinho para atendê-la, acho que a gente ficou aqui umas duas horas e meia. (E2)

No início, demorou um pouco, mas como ele estava bem febril, foi mais rápido depois o atendimento. (E11)

\section{Discussão}

Nas falas dos entrevistados que levaram as crianças ao serviço de urgência e emergência por motivo de febre, identificou-se, essencialmente, a manifestação de grande temor com esse sinal, visto que para eles a febre representava indicativo de doença, muitas vezes, grave. Além disso, as preocupações se intensificaram com sinais e sintomas decorrentes dela, pois podem modificar o comportamento da criança em relação à diminuição da ingesta alimentar e levá-la ao desânimo.

Tem-se a constatação de que a febre faz com que a população procure mais os serviços de saúde, sendo que o tempo entre o início da febre e a procura é muito curto. Em diferentes regiões do mundo, em que o conhecimento e manejo da febre foram investigados, algumas práticas foram desaconselhadas, essencialmente o fato de levar a criança aos serviços de urgência e emergência sem que isso seja realmente necessário ${ }^{17}$.

Em revisão sistemática sobre a febrefobia, a convulsão figura como a principal preocupação, por se tratar de uma experiência assustadora, especialmente para os pais. Entretanto, a administração de antipiréticos não resolve o problema ${ }^{18}$. Recentemente um estudo conseguiu mostrar redução na prevalência de convulsão febril com o uso de paracetamol retal ${ }^{19}$.

Preocupados com a baixa imunidade de crianças, pais ou responsáveis procuram o serviço de urgência e emergência. Sabendo-se que a febre é manifestação necessária para melhor resposta imunológica do hospedeiro, estudo evidenciou que dos 1.097 atendimentos de crianças com febre, somente $0,4 \%$ desses pacientes apresentaram possível evolução para doença invasi$\mathrm{va}^{6}$. O risco de complicações frente a um quadro febril foi encontrado próximo de $3,2 \%{ }^{7}$.
É preciso considerar também os achados de estudos que analisaram os motivos para que pais procurassem os serviços de urgência e emergência em casos de febre. Salienta-se que a indicação de que eles avaliam esse serviço como o local mais adequado, no qual se congrega consulta com especialista, investigação e tratamento da doença de forma rápida e resolutiva. Outro aspecto que contribui para que pais levem filhos(as) aos serviços de urgência e emergência em caso de febre é a facilidade de acesso pela proximidade ou funcionamento em horários em que os serviços de atenção primária estão fechados ${ }^{20,21}$.

Há reconhecimento de que a preocupação excessiva com a febre, pela correlação com situações que nem sempre são reais, acarreta realização de exames desnecessários, uso indiscriminado e inadequado de antibiótico, antitérmicos e analgésicos, bem como sofrimento de pais e crianças $^{22,23}$. Ponto importante a ser compreendido nesta discussão refere-se ao fato de que o uso de antitérmico de maneira profilática não previne complicações².

Relevante observar que no serviço de urgência e emergência não existe vínculo dos profissionais com a família, o que dificulta a avaliação da capacidade dos pais na constatação de possível piora no quadro clínico, permanecendo dúvidas quanto à garantia do acompanhamento das crianças. Além disso, nesses serviços, torna-se mais difícil o seguimento de protocolos de riscos, mesmo que estes representem potencial avanço na abordagem do portador de febre sem foco.

A febre por si não requer tratamento, sendo que os guidelines recomendam que o tratamento deve ser direcionado para o alívio dos sintomas concomitantes ao quadro e não para baixar a temperatura como único objetivo. Além disso, tem-se constatado que não é raro pais oferecerem sub ou superdosagem de medicamentos e utilizarem outras medidas intercaladas que, muitas vezes, são desconfortantes e desnecessárias ${ }^{23}$.

Mesmo frente às evidências em relação à febre, o uso de medicação antipirética é realizada por mais de $50 \%$ dos pais, sendo que a monoterapia ainda é utilizada por cerca de $67 \%$ dos cuidadores $^{24-27}$. A alternância de medicações e/ ou uso concomitante de duas drogas antipiréticas também foram relatados na literatura, assim como encontrado neste estudo. A recomendação é que drogas simultâneas não devem ser utilizadas, devido aos riscos de efeito indesejável e/ou superdosagem. A alternância de duas medicações somente deve ser realizada no caso da persistência dos sintomas que causam desconforto/an- 
gústia na criança, não com objetivo específico de baixar a temperatura, como observado também neste estudo ${ }^{16}$.

Além das medidas medicamentosas, foi constatada nas entrevistas que vários outros métodos eram utilizados para baixar a temperatura. De acordo com resultados de outros estudos, esses métodos são empregados com menor frequência, a saber: banhos mornos, compressas frias, retirada de roupas, aumento da oferta de líquidos, esponjas com álcool ou vinagre e infusão de plantas $^{27-29}$.

Muitas dessas medidas para diminuição da temperatura corporal são desaconselhadas, uma vez que geram mais desconforto do que benefícios à criança. Encontra-se como cuidado relevante, em caso de febre, o aumento na oferta de líquidos, uma vez que a desidratação é uma das principais complicações da febre em crianças menores de 11 meses $^{27}$, o que não foi mencionado pelos entrevistados deste estudo.

Estudo de revisão da literatura que analisou as mudanças de comportamento e conhecimento de pais sobre febre no decorrer de três décadas, demonstrou diferenças pontuais ao longo desses anos, mostrando a permanência de importante lacuna entre conhecimento e comportamento dos responsáveis e as recomendações dos órgãos de saúde ${ }^{30}$, o que evidencia o fenômeno da inércia cultural ${ }^{31}$. Destaca-se que esse fenômeno também atinge profissionais da saúde. Há dados evidenciando que essa condição ocorre por diferentes motivos: muitos pediatras se preocupam principalmente com o desconforto causado na criança e os enfermeiros, com o efeito mais temido: a convulsão ${ }^{32}$.

Em contexto de grande preocupação com a febre em crianças, muitos procuram serviço de saúde por não sentirem segurança em seu mane$\mathrm{jo}^{20}$. Assim, buscam por avaliação de profissional de saúde para reafirmação de que a febre não esteja associada à doença grave e, consequentemente, tranquilizar-se $\mathrm{e}^{22,33}$.

Resgatam-se, assim, as fantasias de pais em relação ao processo saúde e doença de crianças, fazendo-os buscar no outro o que falta em si. $\mathrm{Na}$ visão psicanalítica, quando um fato é fantasiado, pode ser lembrado como se tivesse ocorrido verdadeiramente. Nas fantasias, existe contraste entre realidade psíquica e realidade material. No mundo das neuroses, a realidade psíquica se sobrepõe $\mathrm{e}^{34}$.

Na temática em que responsáveis por crianças com febre manifestaram a compreensão sobre o atendimento nos serviços de urgência e emer- gência, observou-se que consideram a estrutura e o atendimento oferecidos como satisfatórios e revestidos de aparatos que lhes proporcionam segurança. Frente a isso, é preciso considerar que a avaliação do usuário sobre a assistência recebida em serviço de saúde tem como foco essencial o aspecto afetivo da experiência, o que, embora represente um importante quesito da qualidade da assistência ${ }^{35}$ na área da saúde, nem sempre o usuário consegue ter claro seu real significado.

Nessa perspectiva, nas últimas décadas, com a promulgação da nova Constituição Federal, um novo modelo de atenção à saúde vem sendo proposto pela legislação brasileira, o qual defende amplamente a atenção generalista, voltada para a promoção da saúde, na perspectiva interdisciplinar, com vista a superar o modelo biologista, fragmentado, focado em especialidades e tecnologias de alta densidade.

Entretanto, mesmo com todo esforço que vem sendo implementado, observa-se que ainda prevalece entre pais e responsáveis o imaginário de que a assistência recebida no serviço de urgência e emergência, mesmo que em caso de febre sem sinais de localização, ocorre de forma mais efetiva devido aos equipamentos, à proximidade dos recursos da Unidade de Terapia Intensiva, além dos exames complementares que podem ser oferecidos. Estudo realizado na capital de Minas Gerais, Brasil, com mães que levam crianças por motivos não urgentes aos serviços de urgência, também identificou que contribui para isso o fato de serem atendidas por pediatra, haver estrutura física e a possibilidade de realização de exames complementares ${ }^{20}$.

A condição de procurar os serviços de urgência e emergência desnecessariamente, entretanto, tem custo pessoal e social, iniciando pela grande demanda criada, o que compromete o atendimento de situações de maior complexidade, implicando, também, aumento dos gastos dos serviços de saúde. Alternativa a essa condição tem sido a implantação da classificação de risco nos serviços de urgência e emergência, o que, embora tenha representado importante forma de organizar a demanda, nem sempre satisfaz os usuários que consideram o próprio problema como mais relevante quando comparado com a avaliação dos profissionais ${ }^{36}$.

Encontra-se, como fragilidade do estudo, a ausência de análises da compreensão e das condutas de profissionais da saúde frente à situação, evidenciando-se que sua percepção é complementar na definição de intervenções frente a sinais e sintomas de febre. 
Os dados deste estudo contribuem para despertar nos profissionais da saúde a necessidade de ações educativas junto a pais ou responsáveis por crianças, uma vez que o cuidado com a febre, de acordo com as diretrizes, contribui para o menor desgaste deles, maior conforto para as crianças, além do uso racional de antitérmicos e antibióticos.

\section{Considerações finais}

Os entrevistados, pais ou responsáveis por crianças que as levaram ao serviço de urgência e emergência por motivo de febre, atribuem a essa procura grande temor, uma vez que a associam às doenças infecciosas graves, à convulsão e à baixa imunidade. Nos cuidados com a febre, utilizavam medidas medicamentosas, não medicamentosas, bem como a associação de ambas, nem sempre seguindo critéiros apropriados. Quanto ao atendimento nos seviços de urgência e emergência, mostraram-se seguros e confiantes frente à tecnologia de alta densidade existente no cenário investigado.

Urgem, portanto, intervenções com essa população, com vista a tranquilizá-la e apoiá-la, para que se sinta segura no manejo desse sinal corriqueiro entre crianças, uma vez que se trata de manifestação de proteção do organismo e, na maioria das vezes, de evolução benigna. Além disso, são necessários novos estudos, a fim de evidenciar como profissionais da saúde, principalmente aqueles locados na atenção básica, lidam com essa realidade, pois são os principais responsáveis pelo apoio a pais para a condução da situação de forma mais próxima das evidências científicas.

\section{Colaboradores}

PJ Pitoli - Definição do projeto, coleta dos dados, análise dos dados, discussões e redação final do artigo. BK Duarte, AA Fragoso e DG Damaceno - Revisão da literatura, delineamento do projeto, coleta e análise dos dados, discussões e redação final do artigo. MJS Marin - Orientação do projeto, análise dos dados, discussões e redação final do artigo. 


\section{Referências}

1. Magni AM, Scheffer DK, Bruniera P. Antipyretic effect of ibuprofen and dipyrone in febrile children. J Pediatr 2011; 87(1):36-42.

2. NICE Guideline Updates Team (UK). Fever in under 5s: assessment and initial management [internet]. London: National Institute for Health and Care Excellence (UK); 2019 [cited 2019 Nov 26]. Available from: https://www.nice.org.uk/guidance/ng143

3. Razón Behar R. Fiebrefobia. Rev Cuba Pediatr [periódico en internet] 2011 [acceso en 2019 Nov 26]; 83(4):431-441. Disponible en: http://scielo.sld.cu/ pdf/ped/v83n4/ped11411.pdf

4. Mekitarian Filho E, Carvalho WB. Current management of occult bacteremia in infants. J Pediatr 2015; 91(6 Supl. 1):S61-S66.

5. Fernandes TF. Febre não é doença, é um sinal [internet]. São Paulo: Sociedade Brasileira de Pediatria de São Paulo; 2019 [acessado 2019 Maio 26]. Disponível em: http://www.spsp.org.br/site/asp/recomendacoes/ Rec87_2.pdf

6. Bustinduy AL, Chis Ster I, Shaw R, Irwin A, Thiagarajan J, Beynon R, Ladhani S, Sharland M, CABIN network. Predictors of fever-related admissions to a paediatric assessment unit, ward and reattendances in a South London emergency department: the CABIN 2 study. Arch Dis Child 2017; 102(1):22-28.

7. Kool M, Elshout G, Moll HA, Koes BW, van der Wouden JC, Berger MY. Duration of fever and course of symptoms in young febrile children presenting with uncomplicated illness. J Am Board Fam Med 2013; 26(4):445-452.

8. American College of Emergency Physicians Clinical Policies Committee, American College of Emergency Physicians Clinical Policies Subcommittee on Pediatric Fever. Clinical policy for children younger than three years presenting to the emergency department with fever. Ann Emerg Med 2003; 42(4):530-545.

9. Purssell E. Antipyretic use in children: more than just temperature. J Pediatr 2013; 89:1-3.

10. Peetoom KK, Ploum LJ, Smits JJ, Halbach NS, Dinant GJ, Cals JW. Childhood fever in well-child clinics: a focus group study among doctors and nurses. $B M C$ Health Serv Res 2016; 16:240.

11. Patricia C. Evidence-based management of childhood fever: what pediatric nurses need to know. J Pediatr Nurs 2014; 29(4):372-375.

12. Purssell E. Fever in children-a concept analysis. J Clin Nurs 2014; 23(23-24):3575-3582.

13. Cecília M, Minayo M. Amostragem e saturação em pesquisa qualitativa: consensos e controvérsias. Rev Pesqui Qual [periódico na Internet]. 2017 [acessado 2019 mar 23]; 5(7): 1-12. Disponível em: https://editora.sepq.org.br/index.php/rpq/article/view/82/59

14. Braun V, Clarke V. Using thematic analysis in psychology. Qual Research Psychol 2006; 6(2):77-101.

15. Vaismoradi M, Jones J, Turunen H, Snelgrove S. Theme development in qualitative content analysis and thematic analysis. J Nurs Edu Practic 2016; 6(5):100-110.
16. Pitoli PJ, Fragoso A, Duarte BK, Damaceno DG Marin MJS. Febre em crianças: significado atribuído por responsáveis que procuram serviço de urgência e emergência. Atas do $8^{\circ}$ Congresso Ibero-Americano em Investigação Qualitativa: Investigações em Saúde; 2019 jul. 16-19; Lisboa, Portugal. Lisboa: CIAIQ; 2019. p. 985-993.

17. Pérez Polo A, Bartolomé Ferrero A. Actitud y conocimiento de los padres sobre la fiebre. Rev Pediatr Aten Primaria [periódico en internet] 2016 [acceso en 2019 Nov 26]; 18(72):e209-e216. Disponible en: http://scielo.isciii.es/pdf/pap/v18n72/1139-7632-pap-18-720e209.pdf

18. Clericetti CM, Milani GP, Bianchetti MG, Simonetti GD, Fossali EF, Balestra AM, Bozzini MA, Agostoni C, Lava SAG. Systematic review finds that fever phobia is a worldwide issue among caregivers and healthcare providers. Acta Paediatr 2019; 108(8):1393-1397.

19. Lanzlinger D. Reduction in recurrence of febrile seizures with paracetamol use. J Paediatr Child Health 2019; 55(1):116.

20. Rati RM, Goulart LM, Alvim CG, Mota JA. "Criança não pode esperar": a busca de serviço de urgência e emergência por mães e suas crianças em condições não urgentes. Cien Saude Colet 2013; 18(12):36633672 .

21. van Ierland $\mathrm{Y}$, Seiger $\mathrm{N}$, van Veen $\mathrm{M}$, van Meurs $\mathrm{AHJ}$, Ruige M, Oostenbrink R, Moll HA. Self-referral and serious illness in children with fever. Pediatrics [serial on the internet]. 2012 [cited 2019 Nov 26]; 129(3):e643-e651. Available from: https://pediatrics. aappublications.org/content/pediatrics/129/3/e643. full.pdf

22. Bont EG, Loonen N, Hendrix DA, Lepot JM, Dinant GJ, Cals JW. Childhood fever: a qualitative study on parents' expectations and experiences during general practice out-of-hours care consultations. BMC Fam Pract [serial on the internet]. 2015 [cited 2019 Nov 26]; 16:131. Available from: https:// www.ncbi.nlm.nih.gov/pmc/articles/PMC4597376/ pdf/12875_2015_Article_348.pdf

23. Chiappini E, Bortone B, Galli L, Martino M. Guidelines for the symptomatic management of fever in children: systematic review of the literature and quality appraisal with AGREE II. BMJ Open [serial on the internet] 2017; 7(7):e015404. [cited 2019 Nov 26]. Available from: https://bmjopen.bmj.com/content/ bmjopen/7/7/e015404.full.pdf

24. Wallenstein MB, Schroeder AR, Hole MK, Ryan C, Fijalkowski N, Alvarez E, Carmichael SL. Fever literacy and fever phobia. Clin Pediatr (Phila) 2013; 52(3):254-259.

25. Burokiene S, Raistenskis J, Burokaite E, Cerkauskiene $\mathrm{R}$, Usonis V. Factors determining parents' decisions to bring their children to the pediatric emergency department for a minor illness. Med Sci Monit 2017; 23:4141-4148.

26. Figueroa FN, Forero J, León JA, Londoño AC, Echandía CA. Detección, manejo y percepción materna de la fiebre em niños, Cali, Colombia. Rev Fac Med 2012; 60(1):40-49. 
27. Bertille N, Purssell E, Hjelm N, Bilenko N, Chiappini E, Bont EGPM, Kramer MS, Lepage P, Lava SAG, Mintegi S, Sullivan JE, Walsh A, Cohen JF, Chalumeau M. Symptomatic management of febrile illnesses in children: a systematic review and meta-analysis of parents' knowledge and behaviors and their evolution over time. Front Pediatr [serial on the internet] 2018 [cited 2019 Nov 26]; 6:e279. Available from: https:// www.ncbi.nlm.nih.gov/pmc/articles/PMC6183237/ pdf/fped-06-00279.pdf

28. Enarson MC, Ali S, Vandermeer B, Wright RB, Klassen TP, Spiers JA. Beliefs and expectations of Canadian parents who bring febrile children for medical care. Pediatrics [serial on the internet] 2012 [cited 2019 Nov 26]; 130(4):e905-e912. Available from: https:// pediatrics.aappublications.org/content/pediatrics/130/4/e905.full.pdf

29. Nijman RG, Oostenbrink R, Dons EM, Bouwhuis CB, Moll HA. Parental fever attitude and management: influence of parental ethnicity and child's age. Pediatr Emerg Care 2010; 26(5):339-342.

30. Poirier M, Collins E, McGuire E. Fever phobia: a survey of caregivers of children seen in a pediatric emergency department. Clin Pediatr (Phila) 2010; 49(6):530-534.

31. Pereira GL, Tavares NUL, Mengue SS, Dal Pizzol TS. Therapeutic procedures and use of alternating antipyretic drugs for fever management in children. $J \mathrm{Pe}$ diatr 2013; 89(1):25-32.

32. Martins M, Abecasis F. Healthcare professionals approach paediatric fever in significantly different ways and fever phobia is not just limited to parents. Acta Paediatr 2016; 105(7):829-833.

33. Maguire S, Ranmal R, Komulainen S, Pearse S, Maconochie I, Lakhanpaul M, Davies F, Kai J, Stephenson T; RCPCH Fever Project Board. Which urgent care services do febrile children use and why? Arch Dis Child 2011; 96(9):810-816.
34. Silva VCC, Santiago J. "Embelezamento dos fatos" à "cicatriz": uma investigação sobre a fantasia em Freud. Psicol Teor Pesqui [periódico na Internet]. 2017 [acessado 2019 Nov 26]. 33:e33419. Disponível em: https://www.scielo.br/pdf/ptp/v33/0102-3772-ptp33-e33419.pdf

35. Inchauspe JAF, Moura GMSS. Aplicabilidade dos resultados da pesquisa de satisfação dos usuários pela Enfermagem. Acta Paul Enferm 2015; 28(2):177-182.

36. Oliveira JLC, Gatti AP, Barreto MS, Bellucci Junior JA, Góes HLF, Matsuda LM. Acolhimento com classificação de risco: percepções de usuários de uma unidade de pronto atendimento. Texto Contexto Enferm [periódico na Internet] 2017;26(1):e0960014. [acessado 2019 Nov 26]. Disponível em: https://www.scielo. br/pdf/tce/v26n1/pt_0104-0707-tce-26-01-0960014. pdf

Artigo apresentado em 27/04/2020

Aprovado em 03/06/2020

Versão final apresentada em 05/06/2020

Editores-chefes: Maria Cecília de Souza Minayo, Romeu Gomes, Antônio Augusto Moura da Silva 